\title{
HRTEM observation of the metastable phases in Mg-Gd-Y-Zr alloy
}

\author{
Yuya Fukuda ,Tokimasa Kawabata , Kenji Matsuda , Shigeharu Kamado ${ }^{3}$,Yo.Kojima ${ }^{3}$, Susumu Ikeno
}

${ }^{1}$ Graduating student , Toyama University , 3190, Gofuku , Toyama , 930-8555 , Japan

${ }^{2}$ Department of System Engineering for Materials and Science, Faculty of Engineering, Toyama University, 3190,Gofuku,Toyama, 930-8555, Japan

${ }^{3}$ Department of mechanical Engineering, Nagaoka University of Technology, 1630-1 Kamitomioka-machi, Nagaoka, Niigata 940-2188, Japan

\section{Introduction}

Magnesium alloys containing rare earth elements (RE) have good heat resistance and creep resistance. Because their metastable phases remain stable at high temperature [1]. It has been reported that magnesium alloy containing Gd exhibits excellent age hardening [2]. In this study ,Y was added to Mg-Gd binary alloy in order to increase age hardening and reduce the material cost. A lot of studies have been done on the precipitation sequence in Mg-RE alloys, Mg-Gd-Y-Zr alloy however has not been well investigated by HRTEM. The purpose of this study is to investigate transformation in Mg-Gd-Y-Zr alloy by using the high resolution transmission electron microscopy (HRTEM).

\section{Experimental}

The Mg-8.0wt\%Gd-3.7wt\%Y-0.76wt\%Zr (GY85) was used for the present study. The ingot was homogenized at $773 \mathrm{~K}$ for 6 hours, hot rolled to $2 \mathrm{~mm}$ thickness. This sheet was capsulated into pyrex glass tube with Ar gas. Solution heat-treated at $773 \mathrm{~K}$ for 1 hour and quenched in water. The aging treatment was performed at $423 \mathrm{~K}$ and $473 \mathrm{~K}$. Specimens for TEM were prepared by twin-jet electropolishing in a solution 20 percent nitric acid and 80 percent ethanol. These specimens were observed by using HRTEM (TOPCON EM-002B type),operating at $120 \mathrm{kV}$.

\section{Results}

Figure 1(a) and (b) show the selected area diffraction patterns (SADPs) obtained from as quenched and aged GY85 at 473K for 16 hours. It could be seen clear spots in both (a) and (b), and these are Mg matrix reflections. However, it is seen small spots in (b) comparing with (a).These spots were identified as $\beta$, reflections with base-centered orthorhombic (bco) crystal structure $(a=0.640 \mathrm{~nm}, b=2.223, c=0.521 \mathrm{~nm})$ [3] Figure 2 shows a HRTEM image of GY85 aged at $473 \mathrm{~K}$ for 16 hours. The platelet precipitates were observed from the image. It is seen that these precipitates were plates parallel to the $\{10 \overline{1} 0\}$ planes of the matrix. These precipitates were identified as $\beta$ ' precipitate with bco structure from SADPs.

\section{Conclusions}

The platelet precipitates were observed from HRTEM image of GY85 aged at 473K for 16 hours. These precipitates were identified as $\beta$ ' precipitate with bco structure from SADPs. Crystal structure of $\beta$ ' phase in the DY85 alloy agree with another Mg-Re alloys [3].

\section{References}

[1] S.Kamado et al.: Proceedings of the International Conference on Magnesium Alloys and Their Application (1998) 169-174 
[2] S.Kamado et al.: Journal of Japan Institute of Light Metals 42 (1992)

[3] P.J.Apps et al.: Scr Mater 48 (2003) 1023-1028
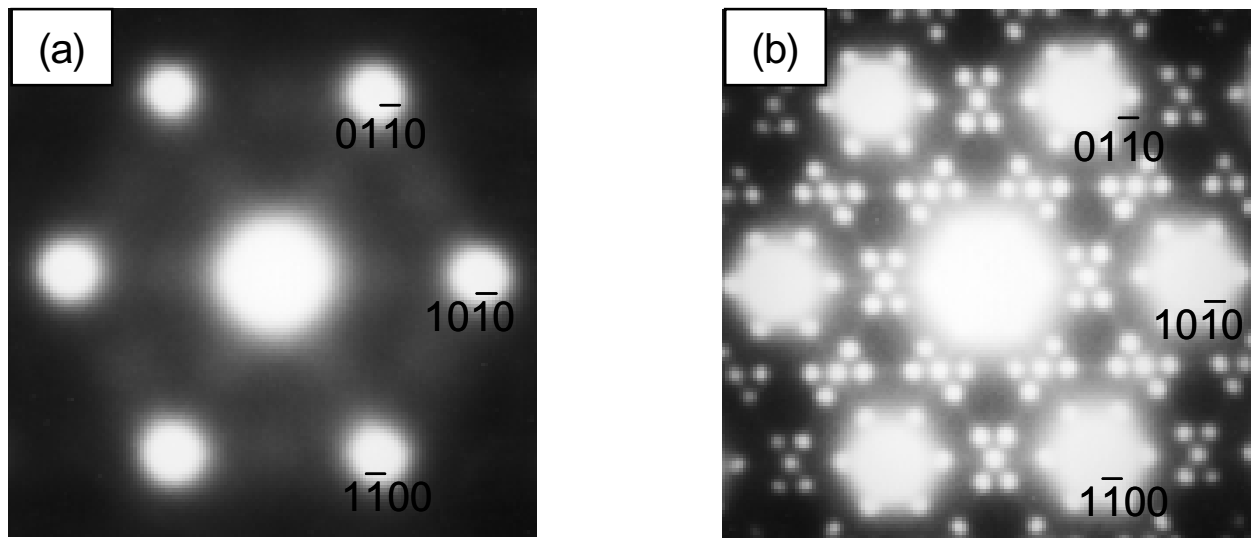

Fig.1 Diffraction patterns of the specimens (a) as quenched and (b) aged at $473 \mathrm{~K}$ for 16 hours (the incident beam direction is parallel to $[0001]_{\mathrm{Mg}}$ ).

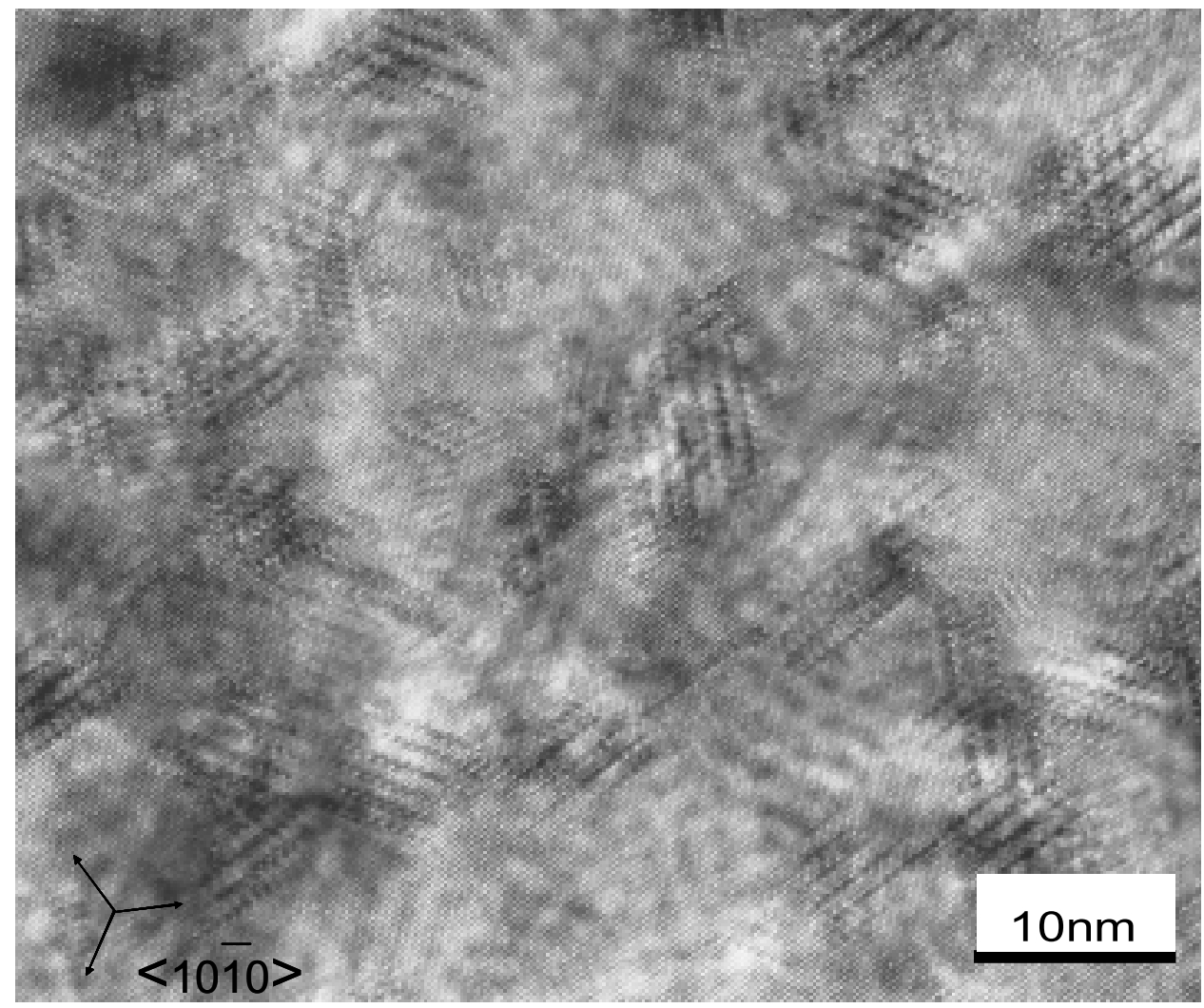

Fig.2 HRTEM image of specimen aged at 473K for 16 hours (the incident beam direction is parallel to $[0001]_{\mathrm{Mg}}$ ). 